\title{
Asteroseismology of rapidly rotating pulsators
}

\author{
W.A. Dziembowski ${ }^{1,2}$ \\ ${ }^{1}$ Warsaw University Observatory, Aleje Ujazdowskie 4, 00-478 Warszawa, Poland \\ email:wd@astrouw.edu.pl \\ ${ }^{2}$ Copernicus Astronomical Center, ul. Bartycka 18, 00-716 Earszawa Poland
}

\begin{abstract}
Oscillation frequencies were determined for a number of rapidly rotating main sequence stars. However, real seismic probing is still ahead of us. I review here tools that we have for modeling pulsation in rotating stars and their potential application to seismic sounding.
\end{abstract}

Keywords. stars: oscillations - stars: rotation

\section{Introduction}

The most important goal of asteroseismology is probing internal rotation and particularly interesting when rotation is rapid. For the upper main sequence stars, we may adopt that beginning with the equatorial velocity of $100 \mathrm{~km} / \mathrm{s}$ rotation should be regarded rapid because then it matters for stellar evolution. With such a definition, most of $\beta$ Cephei and $\delta$ Scuti stars are rapidly rotating pulsators. For the lower main sequence stars, even $10 \mathrm{~km} / \mathrm{s}$ is a large velocity, implying enhanced magnetic activity. So far, assessments of the interior rotation rate were made only for some slowly rotating objects.

\section{The tools}

Interpretation of stellar oscillation spectra requires calculation of evolutionary stellar models and their oscillation properties. For rapidly rotating stars, such complete tools are not yet available.

A cubic (in rotation rate, $\Omega$ ) perturbation theory was developed by Soufi et al.(1998) and Suárez et al. (2006) for uniform and shellular rotation, respectively. In the case of p-modes, more important than including the $\Omega^{3}$ terms is taking into account effects of coupling between close modes of degree differing by 2 . When such effects are included, the treatment may be adequate even somewhat above $100 \mathrm{~km} / \mathrm{s}$ equatorial velocity.

The traditional approximation allows for a strict separation of radial and latitudinal eigenfunctions. It is applicable to slow modes in not too rapidly rotating stars as it ignores effects of centrifugal distortion. Townsend (2005) carried an extensive survey of such modes in main sequence B stars using his generalization of this approximation to nonadiabatic oscillations.

Limitations of the traditional approximation were discussed by Lee \& Saio (1997) and Saio et al. (2006), who compared results obtained with this approximation and a more accurate treatment employing expansion in Legendre function. In their approach the distortion was treated as a linear perturbation. They showed that most of retrograde modes, which are unstable with the traditional approximation, are found with their more accurate treatment.

Accurate description of all effects of rotation was considered so far only to adiabatic oscillations. Reese et al. (2006) developed a method employing Chebyshev and spherical function expansion. The method was originally applied to uniformly rotating polytropic models. Results of application to differentially rotating realistic stellar models were published recently by Reese et al. (2009). Lovekin et al. (2009) solved similar problems with their finite difference 2D code. 


\section{Important applications}

$\beta$ Cephei stars are both most important and promising objects for probing internal rotation. They occur in the mass range, where fast rotation influences stellar evolution up to the end as a supernova explosion or a cooling white dwarf. Estimate of rotation rate in deep interior was made for several objects(see e.g. Dziembowski \& Pamyatnykh, 2008). However, none of them is a rapid rotator. The main advantage of $\beta$ Cep stars is instability of modes sensitive to rotation rate in different layers, including the $\mu$-gradient zone. The main difficulty is that only some of the unstable modes are detected. Mode identification is then crucial but it is not an easy task.

Rich oscillation spectra for four Be stars were found with data from the MOST satellite (Cameron et al. 2008, and references herein). Two sequences of the peaks were identified with unstable high-order prograde g-modes corresponding to $\ell=m=1$ and 2 . This identification led the authors to the conclusion that all four stars rotate with the equatorial velocity close to the maximum (Keplerian) value. This is an important finding, but we should expect more information from these rich spectra. There are chances for determination the frequency spacing between consecutive modes from which we could derive constrains on the internal structure and rotation rate.

For solar-like pulsators, which choose high-order p-modes, even at $20 \mathrm{~km} / \mathrm{s}$ equatorial rotation rate the centrifugal force has a significant impact on theoretical frequency spectra. The asymptotic equality of the $\ell$ and $\ell+2$-mode frequencies implies that the mode coupling is very significant. Multiplets of close low degree modes overlap. Still, it should be possible to recover data needed to obtain some measure of the internal rotation rate.

In lower main stars, rapid rotation is always associated with an enhanced magnetic activity. The related complications of the oscillation spectra are expected large. However, prospects for learning something about subphotospheric rotation and magnetic field in young active stars is so interesting that undertaking the difficult seismic analyzes of such objects is worth the effort.

\section{Acknowledgements}

The work was supported by MNiSW grant number N N203 379636 .

\section{References}

Cameron, C., Saio \& H, Kusching, R. et al. 2008, ApJ, 685, 489

Dziembowski, W. A. \& Pamyatnykh, A. A. 2008, MNRAS, 385, 2061

Lee, U. \& Saio, H. 1997, ApJ, 491, 839

Lovekin, C. C., Deupree, R. G., \& Clement, M. J. 2009, ApJ, , 693, 677L

Ligniéres, F. \& Rieutord, M. 2006, A\& A 455, 607

Reese, D., MacGregor, K. B., Jackson, S. et al. 2009, arXiv0903.4854

Saio, H, Cameron, C.,Kusching, R. et al. 2007, ApJ, 654, 544

Soufi, F., Goupil, M. J., \& Dziembowski, W. A. 1998, A\&A 334, 911

Suárez, J. C., Goupil, M. J. \& Morel, P. 2006, A\&3A 449, 673

Townsend, R. H. D. 2005, MNRAS, 360, 465 